\title{
Downregulation of NFAT5 by RNA interference reduces monoclonal antibody productivity of hybridoma cells
}

\author{
Jihang $\mathrm{Ju}^{1,2}$, Ke Zou ${ }^{1,2}$, Hong $\mathrm{Xie}^{1}$ \\ ${ }^{1}$ Institute of Biochemistry and Cell Biology, Shanghai Institutes for Biological Sciences, Chinese Academy of Sciences, Shanghai \\ 200031, China: ${ }^{2}$ Graduate School of the Chinese Academy of Sciences, Shanghai 200031, China
}

Hybridoma cells display an increase in antibody productivity following exposure to hypertonic conditions. However, the underlying mechanism is not well understood. In the present study, we hypothesize that the nuclear factor of activated T cells 5 (NFAT5)/tonicity enhancer binding protein (TonEBP) functions to increase the antibody productivity of hybridoma cells. NFAT5 is an osmosensitive mammalian transcription factor. However, its ubiquitous expression in various organs that are not bathed in hypertonic milieu suggests that NFAT5 may also regulate cell growth and function under isotonic conditions. In this study, we examined the expression of NFAT5 in hybridoma cells by Western blot analysis, and found that it increased significantly in hypertonic medium. To further define the function of NFAT5 in hybridoma cells, RNA interference technique was used to downregulate the expression of NFAT5 in SGB-8 cells (a hybridoma cell line). In isotonic medium, antibody productivity of hybridoma cells was reduced by downregulation of NFAT5 while cell proliferation was not influenced. The results presented here demonstrate that NFAT5 not only plays an important role in osmotic stress response pathway in hybridoma cells but also is essential for optimal antibody productivity.

Keywords: antibody formation, hybridomas, NFAT transcription factors, RNA interference

Cell Research (2007) 17:264-270. doi: 10.1038/cr.2007.3; published online 20 February 2007

\section{Introduction}

Since the development of hybridoma technology by Kohler and Milstein [1], monoclonal antibodies have found a wide range of uses including in vitro diagnostics, laboratory reagents, and therapeutics. A prerequisite for using monoclonal antibodies is the large-scale cultivation of hybridoma cells under suitable cell culture conditions. Despite the widespread use of hybridoma cell culture for the production of monoclonal antibodies, many fundamental questions about cell physiology and culture conditions remain to be answered. Various approaches have been pursued in the aim of upregulating the antibody productivity of hybridoma cells [2-4]. Hypertonic pressure has been suggested as being an economical solution to increase the

Correspondence: Hong Xie

Tel: +86-21-54921434; Fax: +86-21-54921436;

E-mail: hxie@sibs.ac.cn

Received 10 August 2006; revised 6 November 2006; accepted 10 January 2007; published online 20 February 2007 specific antibody productivity in hybridoma cell cultivation [5]. It has been found that hypertonic pressure preferentially enhances the transcription levels of immunoglobulin (Ig) mRNAs, compared with non-IgG mRNAs. The transcription levels of both heavy chain (HC) and light chain (LC) mRNAs are enhanced as well as antibody productivity [6]. The increase of the post-translational processing rate appears to be commensurate with that of antibody production in response to hyperosmotic stress [7]. Recently, a genome-wide analysis has been performed to investigate the transcriptional response of hybridoma cells towards hypertonic stress using DNA microarrays [8]. In spite of these investigations on how hypertonic stress increases the antibody productivity of hybridoma cells, the detailed mechanism is not yet clearly understood at the basic cellular levels as yet.

In this study, we investigated the expression of nuclear factor of activated T cells 5 (NFAT5; also known as tonicity enhancer binding protein, TonEBP; hereafter referred to as NFAT5) in hybridoma cells and its correlation with monoclonal antibody productivity. NFAT5, a unique mem- 
ber of the NFAT family of transcription factors, was first discovered for its ability to stimulate transcription through its binding to tonicity-responsive enhancer sequences via a Rel-like DNA binding domain [9]. It controls the transcription of genes that function to increase the concentration of intracellular organic osmolytes following exposure to hypertonic environment, including genes coding for aldose reductase (which catalyzes the synthesis of sorbitol), and for transporters of betaine, inositol, and taurine. The accumulation of these compatible organic osmolytes compensates for the cell volume reduction induced by hypertonic stress by allowing the osmotic influx of water into the cell. It is speculated to be an important component of the osmotic-stress response pathway, as it was discovered in renal medulla $[10,11]$. However, the expression of NFAT5 is not limited to the kidney, but in fact is ubiquitous. It is widely expressed during early development and in adulthood, and is found in the brain, heart, liver, and many other organs [12-14]. As the extremes of hypertonicity present within the renal medulla do not appear to exist in these tissues under physiological conditions, the function of NFAT5 may not be limited to regulating transcription in response to hypertonic stress. However, the role of constitutive expression of NFAT5 in most tissues is still undefined $[11,15]$.

In the light of these observations, we hypothesize that NFAT5 may play an important role in monoclonal antibody productivity of hybridoma cells. In the present study, we investigated the expression of NFAT5 in hybridoma cells and found that its expression increased significantly in hypertonic culture medium. Furthermore, the function of NFAT5 in hybridoma cells was evaluated through RNA interference that downregulated the expression of NFAT5. Downregulation of NFAT5 resulted in reduced antibody productivity of hybridoma cells in isotonic medium but did not influence hybridoma cell proliferation. These results demonstrated that NFAT5 is not only a critical component of osmotic response in hybridoma cells, but also essential for optimal production of monoclonal antibodies.

\section{Materials and Methods}

\section{Animals}

8-week-old BALB/c female mice were purchased from Shanghai Laboratory Animal Center, Chinese Academy of Sciences. Animals were kept in conventional conditions and were handled in compliance with Institute of Biochemistry and Cell Biology, Shanghai Institutes for Biological Sciences, Chinese Academy of Sciences (Shanghai, China) guidelines for animal care and use.

\section{Cell culture and reagents}

SGB-7, SGB-8, Hepama-1, and Hepama-2 are all hybridoma cell lines that are produced and kept in our lab. SGB-7 and SGB-8 cells secrete monoclonal antibodies specific for antigens expressed on the surface of Eca-109 cells (an esophageal cancer cell line). Hepama-1 and Hepama-2 cells secrete monoclonal antibodies that specifically bind to cell-surface antigens on SMMC-7721 cells (a hepatocellular carcinoma cell line). Hybridoma and SP2/0 cells were maintained in RMPI 1640 (Life Technologies, Grand Island, NY, USA) supplemented with $10 \%$ heat-inactivated fetal calf serum. Spleen cells were obtained as described [16].

The normal osmolality of the culture medium was $290 \mathrm{mOsm} / \mathrm{kg}$ as assessed by measurement of the freezing point using the cryoscopic osmometer Osmomat 030 from Gonotec (Berlin, Germany). To adjust the medium to higher osmolality (320 to $440 \mathrm{mOsm} / \mathrm{kg}$ ), $\mathrm{NaCl}$ was added into the standard medium.

\section{Reverse transcription-polymerase chain reaction}

Total RNA were prepared with Trizol reagent according to the manufacturer's instruction (Invitrogen, Carlsbad, CA, USA), and were reverse transcribed using oligo (dT) as the primer and M-MLV reverse transcriptase (Promega, Madison, WI, USA). Semi-quantitative polymerase chain reaction (PCR) was carried out to detect the LC of the monoclonal antibody produced by SGB-8 cells (primers: 5'-gat gga get cga cat tgt get cac cca atc tcc-3'; 5'-cat gtc tag aac act cat tcc tgt tga agc tct tg-3'). PCR reaction was performed as the following: denaturation at $94^{\circ} \mathrm{C}$ for 5 min followed by 35 amplification cycles: denaturation at $94{ }^{\circ} \mathrm{C}$ for $30 \mathrm{~s}$, annealing at $55^{\circ} \mathrm{C}$ for $30 \mathrm{~s}$, and extension at $72{ }^{\circ} \mathrm{C}$ for $40 \mathrm{~s}$. The cycles were followed by a final extension at $72{ }^{\circ} \mathrm{C}$ for $10 \mathrm{~min}$. The amplification products were sizefractionated by electrophoresis on a $1 \%$ agarose gel and stained with ethidium bromide. GAPDH (primers: 5'-gtg aag gtc cet gtg aac gg-3'; 5'-gat gca ggg atg atg ttc tg-3') was used as an internal standard.

\section{Western blotting}

For Western blotting analysis of NFAT5, cells were lysed for 30 $\min$ at $4{ }^{\circ} \mathrm{C}$ in a buffer (50 mM Tris, $\mathrm{pH} 7.6,150 \mathrm{mM} \mathrm{NaCl}, 1 \mathrm{mM}$ EDTA, $1 \%$ Triton X-100) containing protease inhibitors: $0.2 \mathrm{mg} / \mathrm{ml}$ aprotinin, $5 \mathrm{mM}$ leupeptin, $1 \mathrm{mM}$ phenylmethylsulfonyl fluoride. The lysates were centrifuged at $14000 \times g$ for $15 \mathrm{~min}$ at $4{ }^{\circ} \mathrm{C}$ and the supernatants were collected. $40 \mathrm{mg}$ of proteins were taken from each sample and loaded in SDS-sample buffer $(50 \mathrm{nM}$ Tris- $\mathrm{HCl}(\mathrm{pH}$ 6.8 ), $2 \% \mathrm{w} / \mathrm{v}$ SDS, $10 \%$ glycerol, $100 \mathrm{mM} \mathrm{DTT}$, and $0.1 \% \mathrm{w} / \mathrm{v}$ bromophenol blue) and size-fractionated by SDS-PAGE on $7.5 \%(\mathrm{w} / \mathrm{v})$ polyacrylamide gels. After being transferred to nitrocellulose transfer membrane (Amersham Pharmacia Biotech, Buckinghamshire, UK), the blots were incubated in blocking buffer (TBS containing 5\% skim milk powder and $0.1 \%$ Tween-20) and probed with diluted rabbit anti-NFAT5 antibody (1:2 000, Affinity Bioreagents, Neshanic station, NJ, USA) for $2 \mathrm{~h}$. The membrane was washed for $30 \mathrm{~min}$ with $0.05 \%$ Tween-20 in PBS and then incubated for $1 \mathrm{~h}$ with horseradish peroxidase-conjugated goat anti-rabbit $\mathrm{IgG}$ antibody solution $(1: 2$ 000, Calibiochem, La Jolla, CA, USA) in blocking buffer. After additional washing steps, the membrane was incubated with DAB staining reagents (Sino-American Biotechnology, Luoyang, China). The relative density of the bands was analyzed by using TotalLab Software (ImageMaster VDS Video Documentation System, Amersham Pharmacia Biotech). The image was captured using a high-performance CCD camera and the system calculated the pixel density of the manually selected band of the immunoblot. NFAT5 from untransfected cells were served as negative control and the actin band provides an internal control for equal protein loading and transfer.

For Western blotting analysis of IgG, supernatants of hybridoma cells were collected from each sample. Equal volumes of supernatants 
were size fractionated by SDS-PAGE on $10 \%(\mathrm{w} / \mathrm{v})$ polyacrylamide gels and then transferred to nitrocellulose membrane. The blots were incubated for $1 \mathrm{~h}$ with horseradish peroxidase-conjugated goat antimouse IgG antibody solution (1:2 000, Jackson ImmunoResearch, PA, USA) in blocking buffer. The membrane was washed and incubated with DAB staining reagents, and then the relative density of the bands was analyzed as described above.

\section{Preparation of siRNA duplexes}

To silence the expression of NFAT5 by RNA interference, we designed three small interfering RNA (siRNA) duplexes targeting different regions of NFAT5 gene. All siRNA sequences were designed according to the manufacturer's recommendations (GenePharma, Shanghai, China) and were subjected to a BLAST search to ensure that they targeted only the desired genes.

RNA duplexes (positive or specific duplex, 5'-cccucucagcaaggguuaudtdt-3' and 5'-auaacccuugcugagagggdtdt-3'; negative control, 5'-uucuccgaacgugucacgutt-3' and 5'-acgugacacguucggagaatt-3') were chemically synthesized and purified using a commercial source (GenePharma).

\section{Transfection}

The day before transfection, hybridoma cells were seeded into six-well plates such that each well was $50-70 \%$ confluent. Transfection of siRNAs was carried out using RNAi-Mate (GenPharma, Shanghai, CHN) with $100 \mathrm{nM}$ siRNAs in final culture medium. Cells were transfected twice with a 24-h interval and cultured for $48 \mathrm{~h}$ before being harvested. Cell lysates were analyzed for NFAT5. The supernatants of hybridoma cells were used to measure IgG by indirect FACS and Western blotting analysis. Cell numbers were counted and calculated by using trypan blue (Sigma-Aldrich, St Louis, MO, USA) exclusion to identify viable cells.

\section{Flow cytometry}

Eca-109 cells were washed twice in Hank's buffer and incubated with hybridoma supernatants $\left(1 \mathrm{~h}\right.$ at $\left.4{ }^{\circ} \mathrm{C}\right)$. After washing, FITC-conjugated anti-mouse IgG (Sigma-Aldrich) was added (15 $\mu \mathrm{g} / \mathrm{ml}, 30 \mathrm{~min}$ at $4{ }^{\circ} \mathrm{C}$ ). Cells were then washed, resuspended in PBS, and analyzed for fluorescent intensity by using FACSCalibur flow cytometer (Becton-Dickinson, San Jose, CA, USA). Control cells were unstained. For each sample, 20000 light-scatter-gated viable cells were collected. The data were analyzed by using FlowJo software (TreeStar, San Carlos, CA, USA). The mean fluorescent intensity was calculated by integrating the fluorescent histograms for the positive cells.

\section{Statistical analysis}

Results are expressed as mean $\pm \mathrm{SD}$, and statistical comparisons were performed using the $\chi^{2}$ test and one-way analysis of variance. $p$-values less than 0.05 were considered statistically significant.

\section{Results}

Expression of NFAT5 in hybridoma cells and its regulation by hypertonic stress

NFAT5 is expressed not only in the renal medulla that is significantly hyperosmolar but also in the brain, thymus, liver, and many other tissues that are not in hypertonic milieu under physiological conditions $[12,17]$. However, its expression in hybridoma cells has not been reported before. As shown in Figure 1A, we used Western blot analysis to detect the NFAT5 protein in splenocytes, SP2/0 cells, and hybridoma cells (SGB-8, SGB-7, Hepama-1, and Hepama-2). As expected, the results show that all of these cells express NFAT5 in isotonic conditions. However, the biological function of NFAT5 under isotonic conditions remains unknown.

NFAT5 is the only known osmosensitive mammalian transcription factor, and we examined its expression under isotonic and hypertonic culture conditions (Figure 1B). SGB-8 and Hepama-1 cells were cultured in standard medium under the isotonic culture condition (RMPI 1640 supplemented with $10 \% \mathrm{FBS}, 290 \mathrm{mOsm} / \mathrm{kg}$ ) or supplemented with $\mathrm{NaCl}$ to increase the osmolality of the culture condition to $380 \mathrm{mOsm} / \mathrm{kg}$. After $48 \mathrm{~h}$, cell lysates were analyzed by Western blot analysis for NFAT5. The results revealed that compared with the isotonic group, culturing

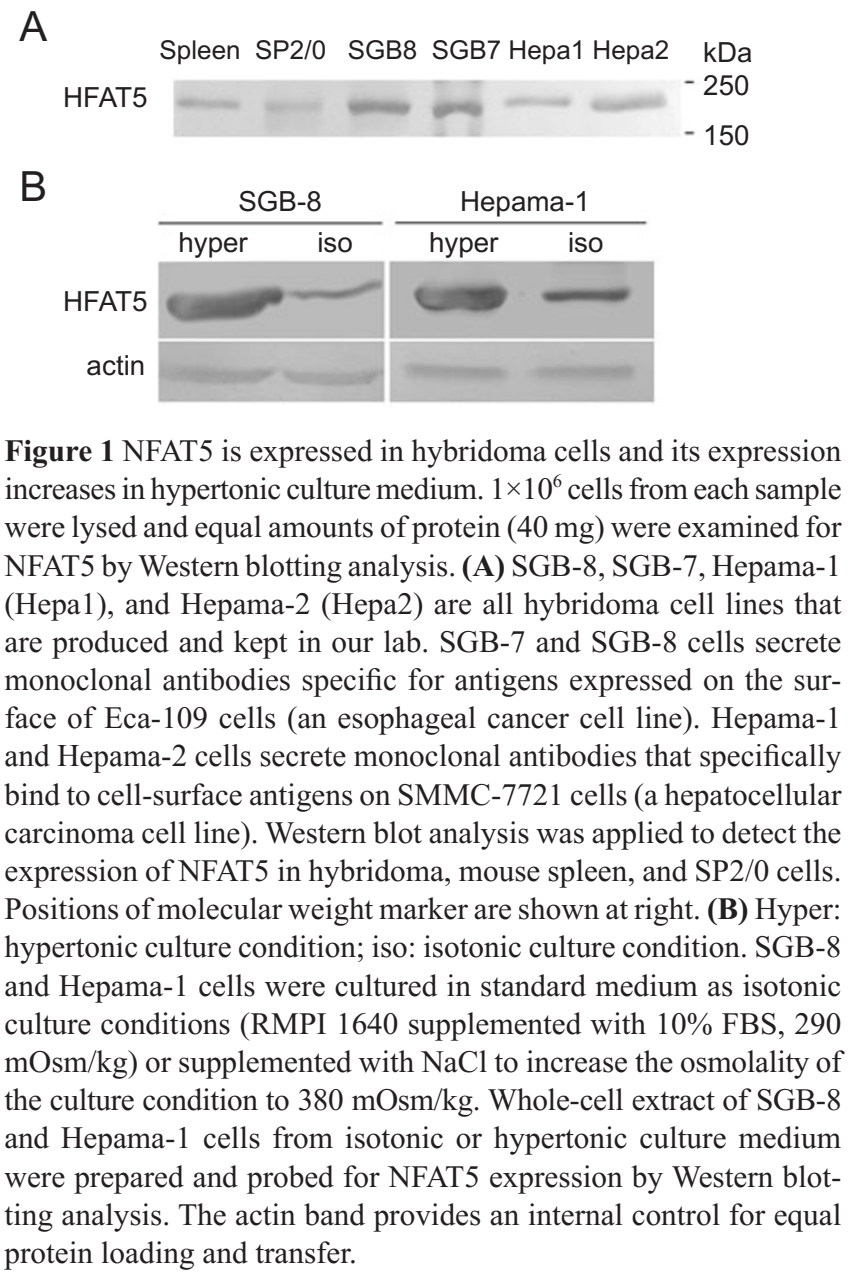


cells in hypertonic medium resulted in increased expression of NFAT5 in SGB-8 and Hepama-1 cells. Densitometric analysis of NFAT5-specific bands on DAB-stained Western blots showed that in hypertonic medium the amount of NFAT5 increased to about three times of that in isotonic medium for both cell lines. Taken together, these data demonstrate that NFAT5 is expressed in hybridoma cells in isotonic conditions and its expression increases significantly following exposure to hypertonic environment.

\section{Downregulation of NFAT5 in SGB-8 cells by RNA inter- ference}

To further define the function of NFAT5 in hybridoma cells, the RNAi technique was used to inhibit the expression of NFAT5 in SGB-8 cells. Three siRNA duplexes, targeting different regions of NFAT5 gene, were synthesized and used to test the efficiency of NFAT5 knockdown. All siRNA sequences were subjected to a BLAST search to ensure their specificity. As shown in Figure 2, one of the siRNA duplexes efficiently reduced the expression of NFAT5 in SGB-8 cells. SGB-8 cells were transfected twice with $100 \mathrm{nM}$ negative control siRNA (-siRNA) or specific siRNA (+siRNA) with a 24-h interval.

Cell lysates were analyzed for NFAT5 by Western blot analysis $48 \mathrm{~h}$ after transfection. The actin band provided an internal control for equal protein loading and transfer. Then the relative density of the scanned NFAT5 bands was determined by using TotalLab software. After the second round of RNA interference, the amount of NFAT5 decreased to $19 \%$ of that in control siRNA transfected cells, and it was reduced to $24 \%$ when the cells were transfected

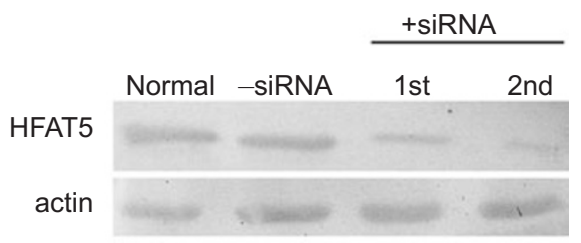

Figure 2 RNA interference downregulates the expression of NFAT5 in SGB-8 cells. SGB-8 cells were transfected twice with $100 \mathrm{nM}$ negative control siRNA (-siRNA) or specific NFAT5 siRNA (+siRNA) with a 24-h interval. 1st: transfected with +siRNA once; 2 nd: transfected with +siRNA twice. Whole-cell extracts were prepared $48 \mathrm{~h}$ after transfection and probed for NFAT5 by Western blot analysis. Cells were incubated in isotonic medium before being harvested. Densitometric analysis showed that after the second round of RNA interference, the amount of NFAT5 decreased to $19 \%$ of that in -siRNA control cells, and it was reduced to $24 \%$ when the cells were transfected only once. NFAT 5 from untransfected cells were used as the negative control and the actin band provides an internal control for equal protein loading and transfer.
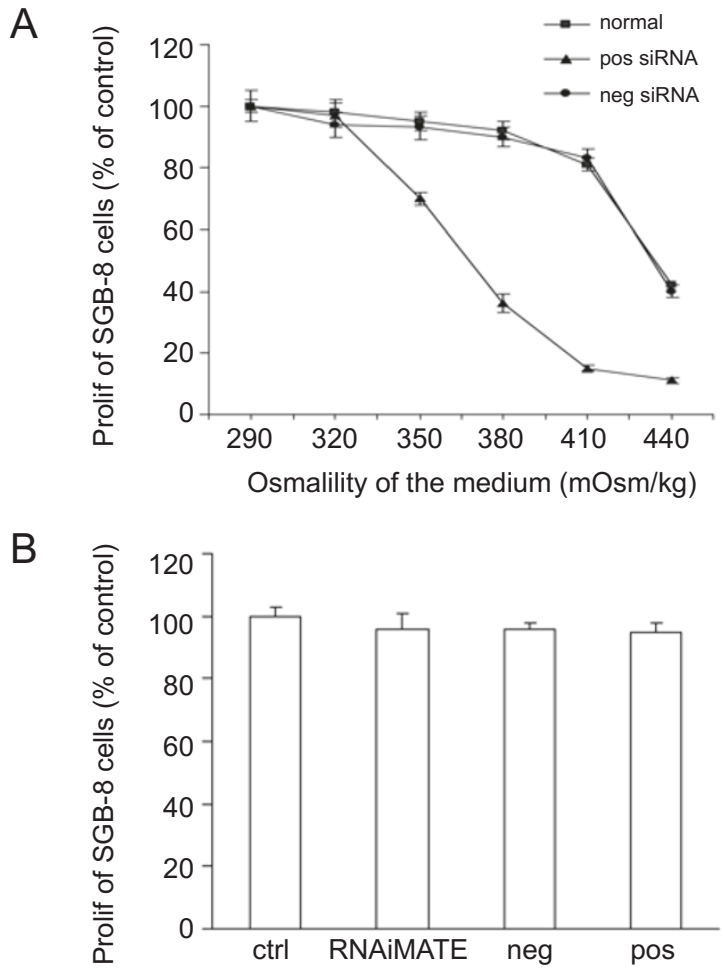

Figure 3 Downregulation of NFAT5 inhibits hybridoma cell proliferation in hypertonic medium, but not in an isotonic environment. (A) Normal, negative control siRNA-treated (neg siRNA) or specific NFAT5 siRNA treated (pos siRNA) SGB-8 cells were cultured in medium of increasing osmolality (290 to $440 \mathrm{mOsm} / \mathrm{kg}$ ). Cell numbers were determined $48 \mathrm{~h}$ later by using trypan blue exclusion to identify viable cells. Cell number of normal SGB- 8 cells in isotonic medium (290 mOsm $/ \mathrm{kg}$ ) was regarded as control. (B) SGB-8 cells were treated with transfection reagent (RNAi-MATE) alone, $100 \mathrm{nM}$ negative control siRNA (neg) or $100 \mathrm{nM}$ specific NFAT5 siRNA (pos), respectively. Cell numbers of each group were determined as described before. The data represent the mean of cell counts from triplicate wells.

only once. Thus in the later part of this study, SGB-8 cells were transfected twice in order to downregulate NFAT5 as much as possible. These results demonstrate that the designed specific siRNA effectively downregulates the expression of NFAT5 in SGB-8 cells.

Downregulation of NFAT5 inhibits hybridoma cell proliferation in hypertonic medium, but not in isotonic environment

To further confirm the downregulation of NFAT5 by the designed specific siRNA and to determine whether the downregulation of NFAT5 influences the proliferation of hybridoma cells, cell proliferation was investigated in iso- 
tonic and hypertonic culture medium. As shown in Figure 3A, normal, control siRNA treated SGB-8 (-siRNA-SGB8 ) and specific siRNA treated SGB-8 (+siRNA-SGB-8) cells were cultured in media of increasing osmolality (290 to $440 \mathrm{mOsm} / \mathrm{kg}$ ). To adjust osmolality of the culture medium, $\mathrm{NaCl}$ was added into the standard medium. Cell were cultured for $48 \mathrm{~h}$, and cell numbers were determined $48 \mathrm{~h}$ later by using trypan blue exclusion to identify viable cells. The proliferation of normal and control siRNAtreated SGB-8 cells was not obviously inhibited until the osmolality of the medium was raised to more than 400 $\mathrm{mOsm} / \mathrm{kg}$. However, with the increasing osmolality of the culture medium, the proliferation of specific siRNA-treated SGB-8 cells was severely inhibited. When the osmolality was increased to $380 \mathrm{mOsm} / \mathrm{kg}$, the proliferation rate of the cells was inhibited by $>65 \%$. These results demonstrate that NFAT5 is essential for the proliferation of SGB-8 cells in hypertonic environment and it is downregulated by the designed + siRNA. However, the scenario appeared to be different under isotonic conditions (Figure 3B). SGB-8 cells were treated with RNAi-Mate (transfection reagent) alone, control siRNA or specific siRNA, respectively. The cells were then cultured in standard medium as isotonic culture conditions. Compared with control siRNA and RNAi-Mate groups, SGB-8 cell proliferation of specific siRNA group was not impaired by downregulation of NFAT5. Taken together, these results demonstrate that after the downregulation of NFAT5, hybridoma cell proliferation was inhibited in hypertonic medium, but not affected in an isotonic environment.

Downregulation of NFAT5 results in reduced antibody productivity of hybridoma cells in isotonic medium

To determine the monoclonal antibody productivity of SGB-8 cells following the downregulation of NFAT5, FACS, Western blotting, and semi-quantitative reverse transcription (RT)-PCR were applied. SGB-8 cells were transfected with control or specific siRNA as described above, then were raised in isotonic medium for $48 \mathrm{~h}$ before being analyzed. The supernatants of hybridoma cells were collected and used for FACS and Western blotting analysis. $1 \times 10^{6}$ cells were harvested and the total RNAs were prepared to detect the LC message of the monoclonal antibody in SGB-8 cells by semi-quantitative RT-PCR (Figure 4A). GAPDH band was used as an internal control. Densitometric analysis showed that when the specific siRNA was used, the amount of LC message decreased to $<50 \%$ of that in control cells. Indirect FACS was performed to analyze IgG in the supernatants. As shown in Figure 4B, Eca-109 cells (SGB-8 cells secrete a kind of monoclonal antibody specific for an antigen expressed on the surface of Eca-109 cells) were incubated with hybridoma supernatants. After wash-
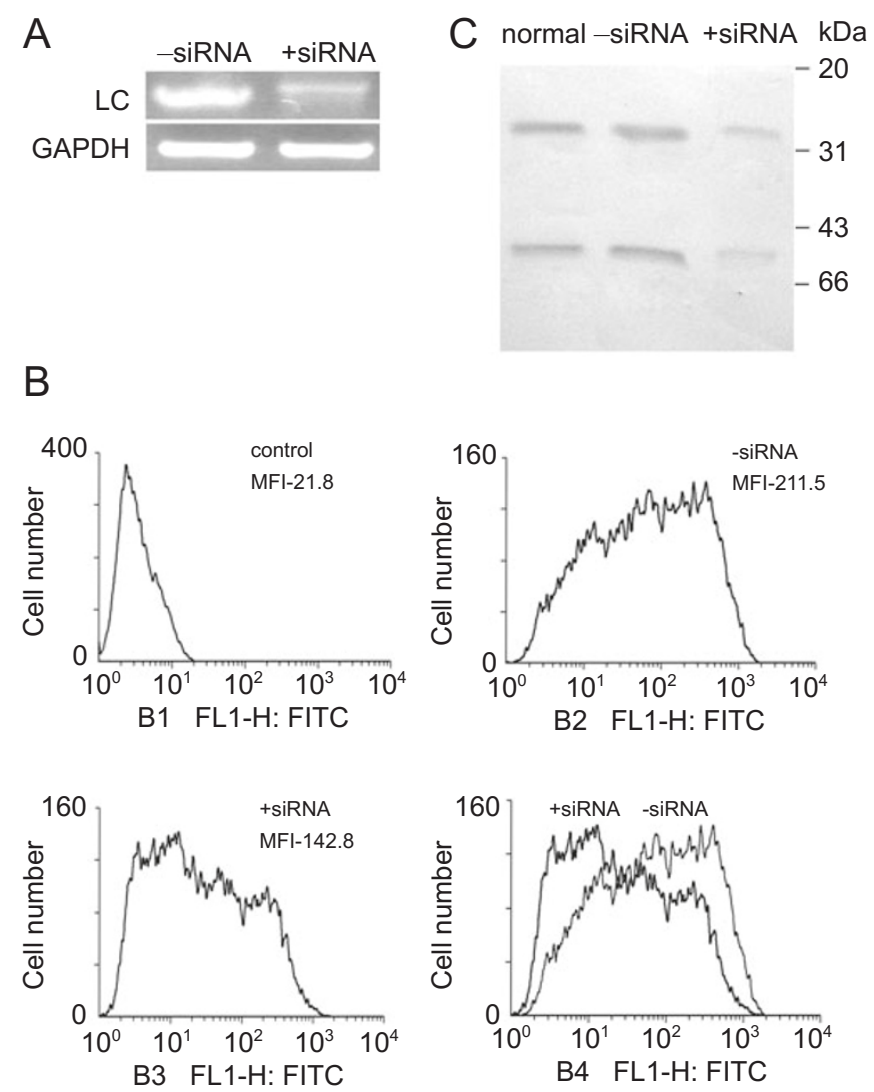

Figure 4 Downregulation of NFAT5 results in reduced antibody productivity of hybridoma cells. SGB- 8 cells were transfected with negative control siRNA (-siRNA) and specific NFAT5 siRNA $(+$ siRNA) twice with a 24-h interval, then raised in isotonic medium for $48 \mathrm{~h}$ before being analyzed. The supernatants were collected and used for FACS and Western blotting analysis. (A) $1 \times 10^{6}$ cells were collected and the total RNA was prepared to detect the LC message of the monoclonal antibody in SGB-8 cells by semi-quantitative RTPCR. GAPDH band was used as the internal control. Densitometric analysis showed that when +siRNA was used, the amount of LC message of the monoclonal antibody decreased to $<50 \%$ of that in control cells. (B) Eca-109 cells (SGB-8 cells secrete a monoclonal antibody specific for an antigen expressed on the surface of Eca-109 cells) were incubated with hybridoma supernatants. After washing, FITC-conjugated secondary antibody was added. Then cells were analyzed by using FACS for the fluorescent intensity. The mean fluorescent intensity (MFI) of the positive cells is indicated in the upper right portion of each FACS plot. In the control group, Eca 109 cells were not treated with antibodies. Compared with the-siRNA group, the MFI of the +siRNA group is reduced significantly $(p<0.001)$. The experiments were performed three times, and a representative set of data is shown. (C) Equal volumes of the supernatants from each sample were fractionated by SDS-PAGE, transferred to nitrocellulose membranes, and probed for the LCs and the HCs of the monoclonal antibody. Densitometric analysis showed that the amount of monoclonal antibody in supernatants of + siRNA group decreased to $<45 \%$ of that in - siRNA control supernatants. 
ing, FITC-conjugated secondary antibodies were added. Then cells were analyzed using FACS for the fluorescent intensity. Compared with the control siRNA group, the mean fluorescent intensity of the specific NFAT5 siRNA group was reduced significantly $(p<0.001 ; n=3$ independent experiments). To further evaluate whether the concentration of the monoclonal antibody in supernatants of hybridoma cells was reduced following the downregulation of NFAT5, the monoclonal antibody in the supernatants was measured by using Western blotting analysis (Figure 4C). Equal volumes of the supernatants from each sample were fractionated by SDS-PAGE, then transferred to nitrocellulose membranes, and probed for the LCs and the HCs of the monoclonal antibody with horseradish peroxidase-conjugated goat anti-mouse $\mathrm{IgG}$ antibody. Densitometric analysis showed that when SGB-8 cells were transfected with the specific NFAT5 siRNA, the amount of monoclonal antibody in the supernatant decreased to $<45 \%$ of that in the supernatant of control siRNA-treated SGB-8 cells. Therefore after the downregulation of NFAT5 in SGB-8 cells, the concentration of monoclonal antibody in the supernatant was reduced. These data demonstrate that in isotonic culture medium, downregulation of NFAT5 results in reduced monoclonal antibody productivity of hybridoma cells.

\section{Discussion}

NFAT5, the only known osmosensitive transcription factor, is expressed in many tissues bathed in either isotonic or hypertonic environment in vivo $[12,15]$. A genome database search reveals that Rel-like transcription factors are present in Drosophila and mammals but not in Caenorhabditis elegans, yeast, and plants [18]. NFAT5 plays an important role in allowing cell to adapt to hypertonic environment. In Madin-Darby canine kidney cells, hypertonicity increases the abundance of NFAT5, contributing to increased transcription of downstream osmoprotective genes [19]. Hypertonicity stabilizes NFAT5 mRNA which depends on the presence of the 5 '-untranslated region (UTR) [13]. As expected, NFAT5 is expressed in hybridoma cells in isotonic culture medium and its expression increases significantly in hypertonic environment. However, the function of NFAT5 under isotonic conditions remains to be investigated. It may not be limited to regulating transcription in response to hypertonic stress, as occurs in the renal medulla. One homolog of NFAT5 named MESR1 has been found in Drosophila (GenBank Accession No. AF195496), which was identified as a modifier of RAS1 signaling involved in eye development [20].

Some previous studies have demonstrated the effect of hypertonic stress on immune function. The relevance of osmotic stress to the function of $\mathrm{T}$ cells or peripheral blood mononuclear cells was first demonstrated in ex vivo studies, showing that proliferation or cytokine production is enhanced upon culture in hypertonic medium [21-23]. In T cells, NFAT 5 controls the osmotic stress-induced expression of several cytokines, including tumor-necrosis factor, interleukin-2, and lymphotoxin- $\beta$ [24, 25]. The release of and response to ATP plays a key role in the mechanism through which hypertonic stress regulates the function of T cells [26]. NFAT5 is also necessary for optimal T-cell development in vivo and allows for optimal cell growth ex vivo under conditions associated with osmotic stress [27]. Furthermore, William Go and his co-workers [21, 28] measured lymphoid tissue osmolality directly by using a vapor pressure osmometer and they found that in contrast to brain and lung, lymphoid tissues were significantly hypertonic relative to serum. These results suggested that NFAT5, the osmosensitive transcription factor, functions to optimize lymphocyte function in hypertonic lymphoid microenvironment. In the present study, we investigated the function of NFAT5 in hybridoma cells by downregulating NFAT5 via effective RNA interference. The specific siRNA duplexes that we designed efficiently reduced the expression of NFAT5 in hybridoma cells. The data revealed that downregulation of NFAT5 in hybridoma cells results in decreased antibody productivity in isotonic medium. In addition, the antibody productivity of hybridoma cells can be elevated by 40 to 50 percent in hypertonic culture medium [6], and the expression of NFAT5 in hybridoma cells increases significantly in hypertonic culture medium. These results indicate that optimal antibody productivity of hybridoma cells is dependent on the function of NFAT5.

In summary, our results presented here revealed new insight into the biologic function of the NFAT5 transcription factor by demonstrating that NFAT5 is essential for optimal antibody productivity of hybridoma cells in isotonic conditions. A more detailed understanding of mechanisms by which NFAT5 affects the production of monoclonal antibodies may lead to new strategies to modulate lymphocyte function for clinically beneficial purposes.

\section{Acknowledgments}

This project was supported by the Major State Basic Research Program of China (2002AA2Z3329). We thank Drs LX Guo and YB Chen for thoughtful discussion during the course of study, and Z Tan for technical assistance with flow cytometry.

\section{References}

1 Kohler G, Milstein C. Continuous cultures of fused cells secreting antibody of predefined specificity. Nature 1975; 256:495-497.

2 deZengotita VM, Schmelzer AE, Miller WM. Characterization 
of hybridoma cell responses to elevated $\mathrm{pCO}(2)$ and osmolality: intracellular $\mathrm{pH}$, cell size, apoptosis, and metabolism. Biotechnol Bioeng 2002; 77:369-380.

3 Balcarcel RR, Stephanopoulos G. Rapamycin reduces hybridoma cell death and enhances monoclonal antibody production. Biotechnol Bioeng 2001; 76:1-10.

4 Heilmann K, Groth T, Behrsing O, et al. The influence of the chemical composition of cell culture material on the growth and antibody production of hybridoma cells. J Biotechnol 2005; 115:291-301.

5 Hencsey Z, Fizil A, Inzelt-Kovacs M, Veszely G, Bankuti L. Effect of medium composition on hybridoma growth and antibody production. Acta Microbiol Immunol Hung 1996; 43:359-370.

6 Lee MS, Lee GM. Hyperosmotic pressure enhances immunoglobulin transcription rates and secretion rates of KR12H-2 transfectoma. Biotechnol Bioeng 2000; 68:260-268.

7 Sun Z, Zhou R, Liang S, McNeeley KM, Sharfstein ST. Hyperosmotic stress in murine hybridoma cells: effects on antibody transcription, translation, posttranslational processing, and the cell cycle. Biotechnol Prog 2004; 20:576-589.

8 Shen D, Sharfstein ST. Genome-wide analysis of the transcriptional response of murine hybridomas to osmotic shock. Biotechnol Bioeng 2006; 93:132-145.

9 Miyakawa H, Woo SK, Dahl SC, Handler JS, Kwon HM. Tonicity-responsive enhancer binding protein, a rel-like protein that stimulates transcription in response to hypertonicity. Proc Natl Acad Sci USA 1999; 96:2538-2542.

10 Burg MB, Kwon ED, Kultz D. Regulation of gene expression by hypertonicity. Annu Rev Physiol 1997; 59:437-455.

11 Ho SN. Intracellular water homeostasis and the mammalian cellular osmotic stress response. J Cell Physiol 2006; 206:9-15.

12 Maouyo D, Kim JY, Lee SD, Wu YH, Woo SK, Kwon HM. Mouse TonEBP-NFAT5: expression in early development and alternative splicing. Am J Physiol-Renal Physiol 2002; 282: F802-F809.

13 Cai Q, Ferraris JD, Burg MB. High $\mathrm{NaCl}$ increases TonEBP/ OREBP mRNA and protein by stabilizing its mRNA. Am J Physiol Renal Physiol 2005; 289:F803-F807.

14 Loyher ML, Mutin M, Woo SK, Kwon HM, Tappaz ML. Transcription factor tonicity-responsive enhancer-binding protein (TonEBP) which transactivates osmoprotective genes is expressed and upregulated following acute systemic hypertonicity in neurons in brain. Neuroscience 2004; 124:89-104.
15 Zhang Z, Ferraris JD, Brooks HL, Brisc I, Burg MB. Expression of osmotic stress-related genes in tissues of normal and hyposmotic rats. Am J Physiol Renal Physiol 2003; 285:F688F693.

16 Phepherd P, Dean C. Monoclonal Antibodies. Oxford: Oxford University Press, 2000.

17 Lopez-Rodriguez C, Aramburu J, Rakeman AS, Rao A. NFAT5, a constitutively nuclear NFAT protein that does not cooperate with Fos and Jun. Proc Natl Acad Sci USA 1999; 96:7214-7219.

18 Riechmann JL, Heard J, Martin G, et al. Arabidopsis transcription factors: genome-wide comparative analysis among eukaryotes. Science 2000; 290:2105-2110.

19 Woo SK, Dahl SC, Handler JS, Kwon HM. Bidirectional regulation of tonicity-responsive enhancer binding protein in response to changes in tonicity. Am J Physiol Renal Physiol 2000; 278: F1006-F1012.

20 Huang AM, Rubin GM. A misexpression screen identifies genes that can modulate RAS1 pathway signaling in Drosophila melanogaster. Genetics 2000; 156:1219-1230.

21 Go WY, Liu XB, Roti MA, Liu F, Ho SN. NFAT5/TonEBP mutant mice define osmotic stress as a critical feature of the lymphoid microenvironment. Proc Natl Acad Sci USA 2004; 101:1067310678.

22 Junger WG, Liu FC, Loomis WH, Hoyt DB. Hypertonic saline enhances cellular immune function. Circ Shock 1994; 42:190196.

23 Shapiro L, Dinarello CA. Osmotic regulation of cytokine synthesis in vitro. Proc Natl Acad Sci USA 1995; 92:12230-12234.

24 Macian F. NFAT proteins: key regulators of T-cell development and function. Nat Rev Immunol 2005; 5:472-484.

25 Lopez-Rodriguez C, Aramburu J, Jin L, Rakeman AS, Michino M, Rao A. Bridging the NFAT and NF-kappaB families: NFAT5 dimerization regulates cytokine gene transcription in response to osmotic stress. Immunity $2001 ; \mathbf{1 5 : 4 7 - 5 8 .}$

26 Loomis WH, Namiki S, Ostrom RS, Insel PA, Junger WG. Hypertonic stress increases $\mathrm{T}$ cell interleukin-2 expression through a mechanism that involves ATP release, P2 receptor, and p38 MAPK activation. J Biol Chem 2003; 278:4590-4596.

27 Trama J, Go WY, Ho SN. The osmoprotective function of the NFAT5 transcription factor in T cell development and activation. J Immunol 2002; 169:5477-5488.

28 Bird L. Lymphocyte responses - NFAT5: helping out in stressful times. Nat Rev Immunol 2004; 4:581. 